\title{
Thermoelectric performance enhancement of the cost-effective phosphide
} $\mathrm{ZnCu}_{2} \mathbf{P}_{8}$

\author{
Justin Mark, ${ }^{\text {a }}$ Takao Moria,b,*
}

${ }^{a}$ International Center for Materials Nanoarchitectonics (WPI-MANA), National Institute for Materials Science (NIMS), Tsukuba, Ibaraki 305-0044, Japan

${ }^{b}$ Graduate School of Pure and Applied Science, University of Tsukuba, Tsukuba, Ibaraki 3058671, Japan

*Corresponding author, e-mail: mori.takao@nims.go.jp

\section{Supporting Information}


Table S1. Lattice parameters determined from Rietveld refinement for $\mathrm{Zn}_{1-x} \mathrm{Cu}_{2+x} \mathrm{P}_{8}(x=-0.25,0$, $0.1,0.25)$ and $\mathrm{Zn}_{0.75} \mathrm{Cu}_{2} \mathrm{P}_{8}$

\begin{tabular}{|c|c|c|c|}
\hline Nominal Composition & $\boldsymbol{a}(\boldsymbol{\AA})$ & $\boldsymbol{b}(\boldsymbol{\AA})$ & $\boldsymbol{c}(\boldsymbol{\AA})$ \\
\hline $\mathrm{Zn}_{0.75} \mathrm{Cu}_{1.25} \mathrm{P}_{8}$ & $11.097(1)$ & $9.644(3)$ & $7.5101(7)$ \\
\hline $\mathrm{ZnCu}_{2} \mathrm{P}_{8}$ & $11.124(1)$ & $9.639(3)$ & $7.5188(7)$ \\
\hline $\mathrm{ZnCu}_{2} \mathrm{P}_{8}\left(\mathrm{reported}^{1}\right)$ & $11.108(2)$ & $9.647(2)$ & $7.527(1)$ \\
\hline $\mathrm{Zn}_{0.9} \mathrm{Cu}_{2.1} \mathrm{P}_{8}$ & $11.120(1)$ & $9.6407(2)$ & $7.5187(5)$ \\
\hline $\mathrm{Zn}_{0.75} \mathrm{Cu}_{2.25} \mathrm{P}_{8}$ & $11.106(2)$ & $9.6399(4)$ & $7.517(1)$ \\
\hline $\mathrm{Zn}_{0.75} \mathrm{Cu}_{2} \mathrm{P}_{8}$ & $11.102(1)$ & $9.6393(2)$ & $7.5167(5)$ \\
\hline
\end{tabular}
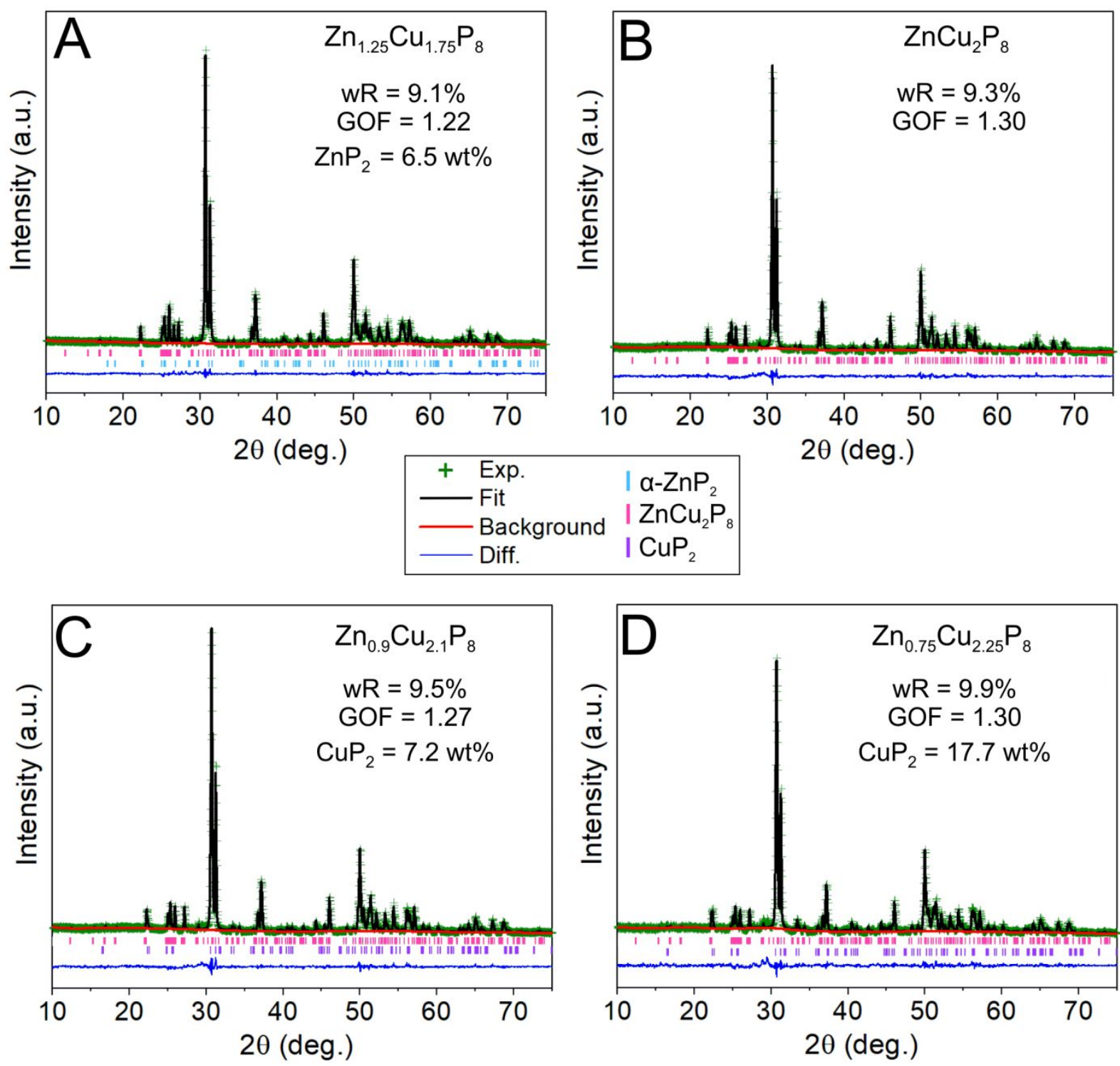

Figure S1. Rietveld refinement for sintered samples of (A) $\mathrm{Zn}_{1.25} \mathrm{Cu}_{1.75} \mathrm{P}_{8}$, (B) $\mathrm{ZnCu}_{2} \mathrm{P}_{8}$, (C) $\mathrm{Zn}_{0.9} \mathrm{Cu}_{2.1} \mathrm{P}_{8}$, and (D) $\mathrm{Zn}_{0.75} \mathrm{Cu}_{2.25} \mathrm{P}_{8}$. Respective secondary phases included in each refinement are listed in each plot. 


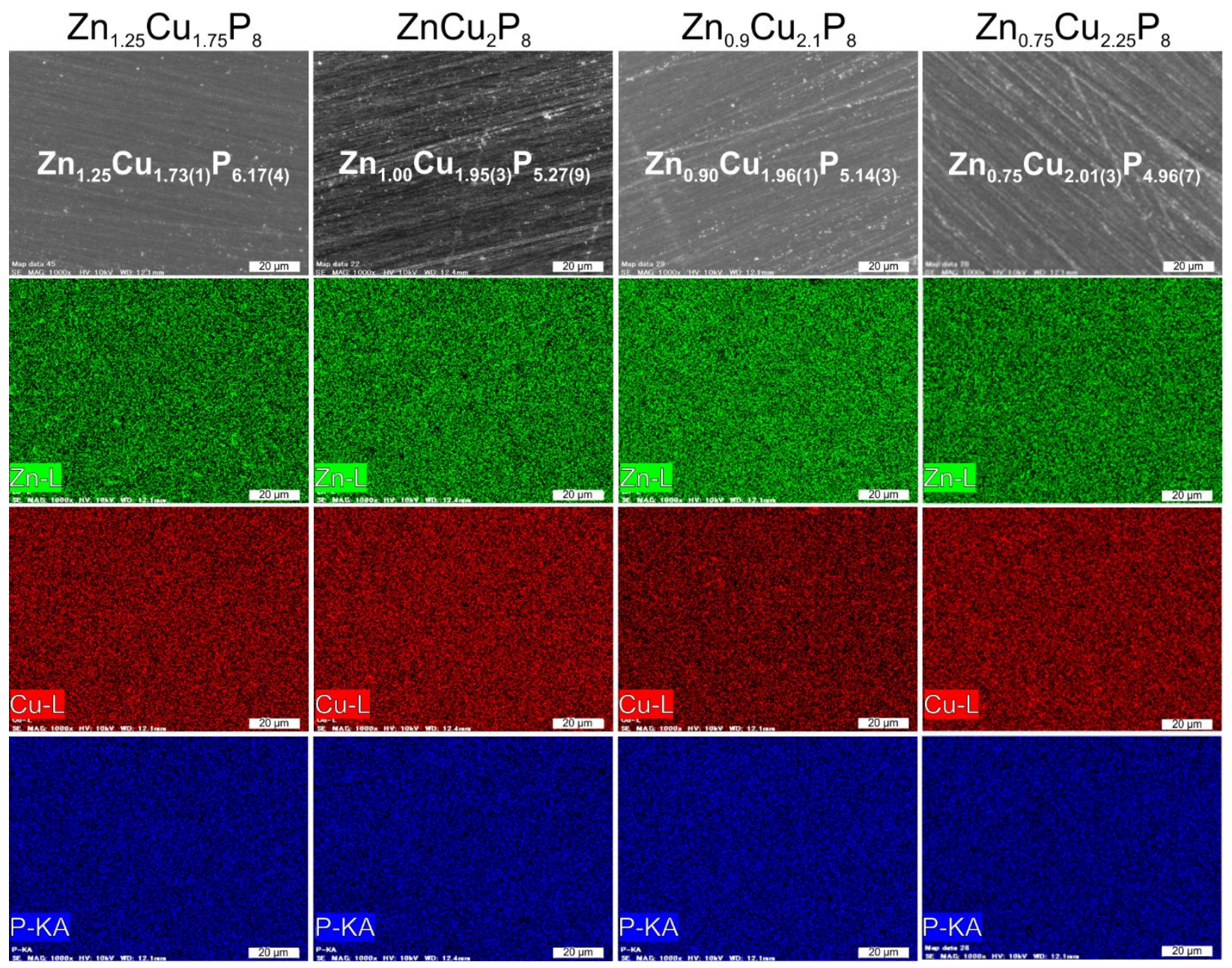

Figure S2. SEM-EDS images of $\mathrm{Zn}_{1-x} \mathrm{Cu}_{2+x} \mathrm{P}_{8}(x=-0.25,0,0.1,0.25)$ samples. Nominal and experimentally determined compositions are shown at the top. Compositions have been normalized to nominal zinc composition. 


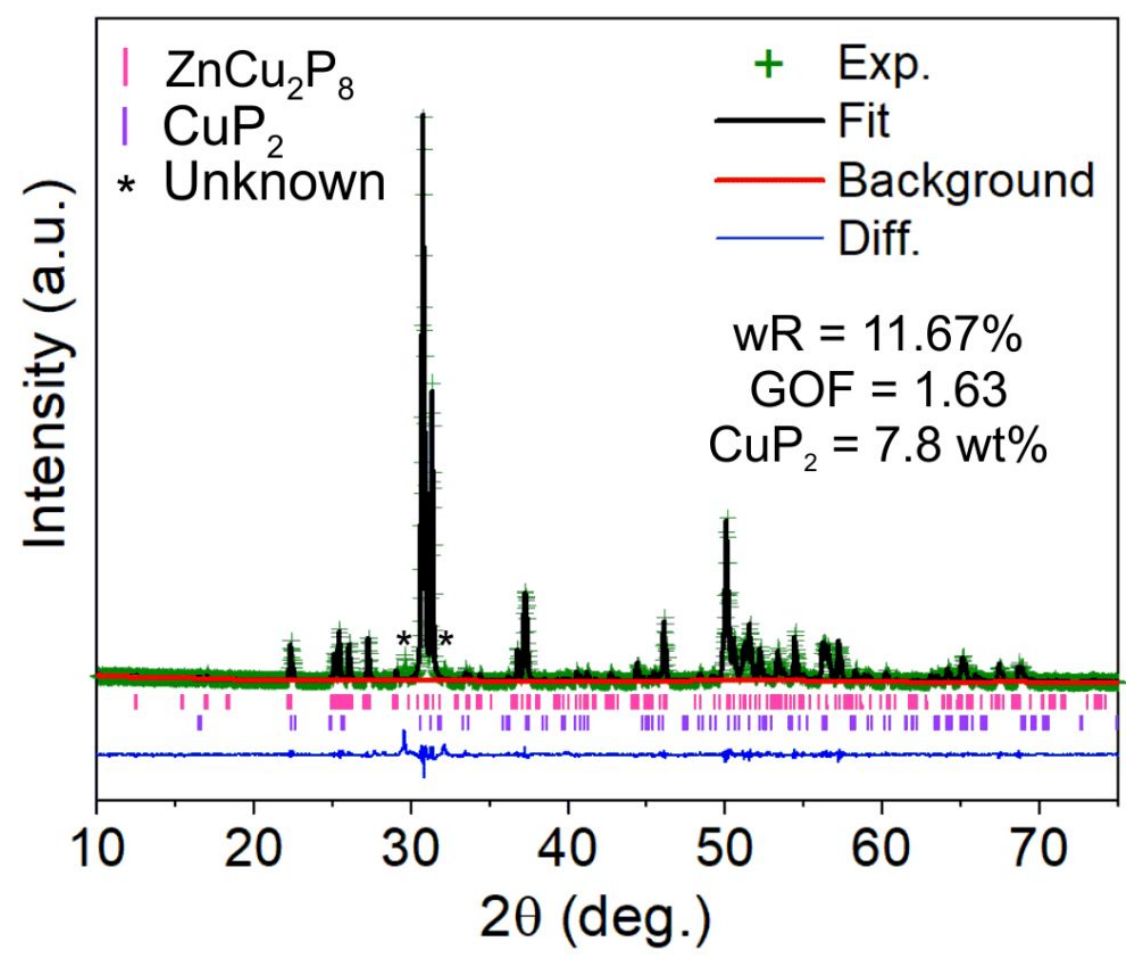

Figure S3. Rietveld refinement for a sintered sample of $\mathrm{Zn}_{0.75} \mathrm{Cu}_{2} \mathrm{P}_{8}$. 

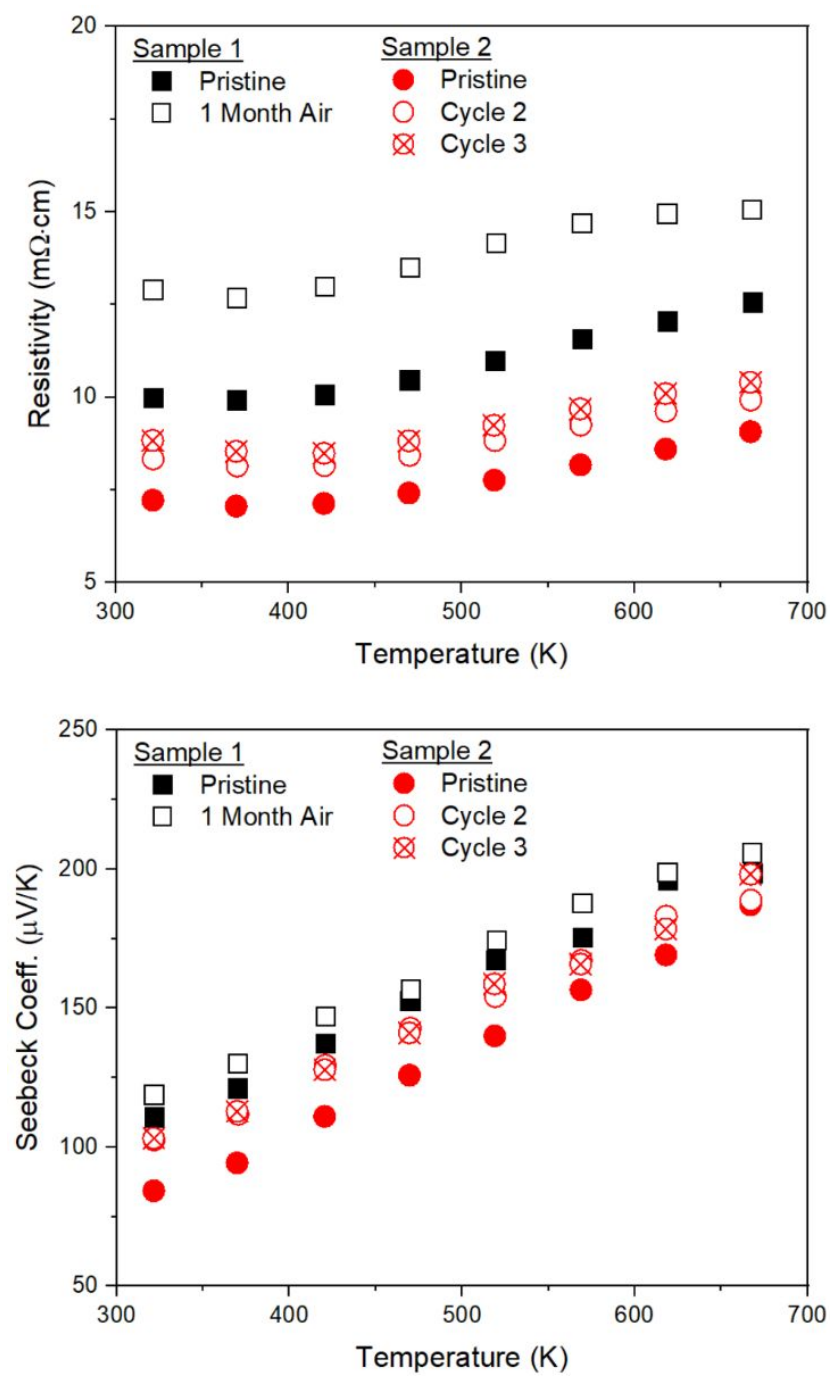

Figure S4. Multiple measurements of electrical resistivity (top) and Seebeck coefficient (bottom) of samples of nominal $\mathrm{Zn}_{0.75} \mathrm{Cu}_{2} \mathrm{P}_{8}$ composition illustrating long term stability of properties. 

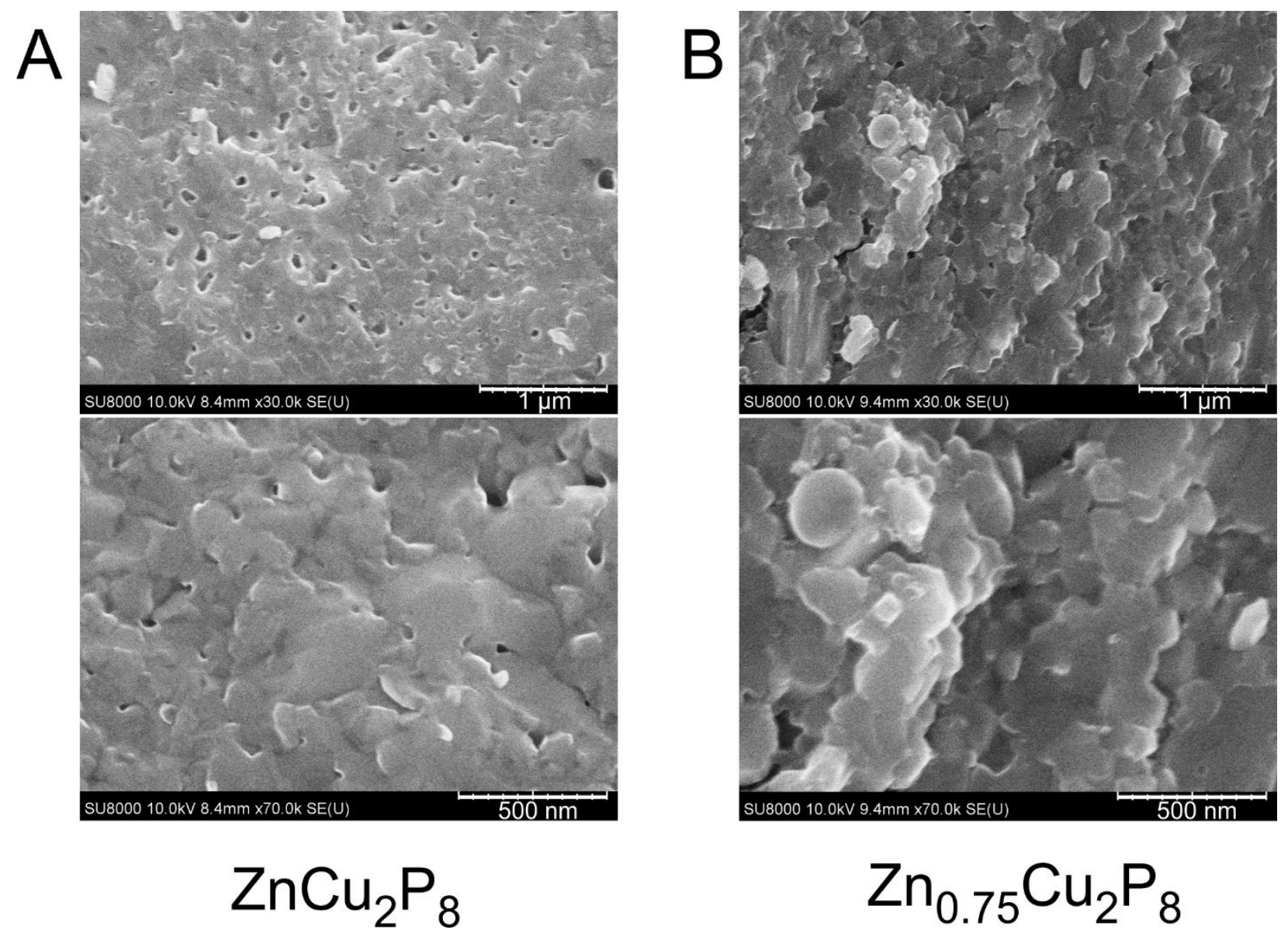

Figure S5. SEM images of fractured samples of nominal composition (A) $\mathrm{ZnCu}_{2} \mathrm{P}_{8}$ and (B) $\mathrm{Zn}_{0.75} \mathrm{Cu}_{2} \mathrm{P}_{8}$ showing difference in microstructure. 


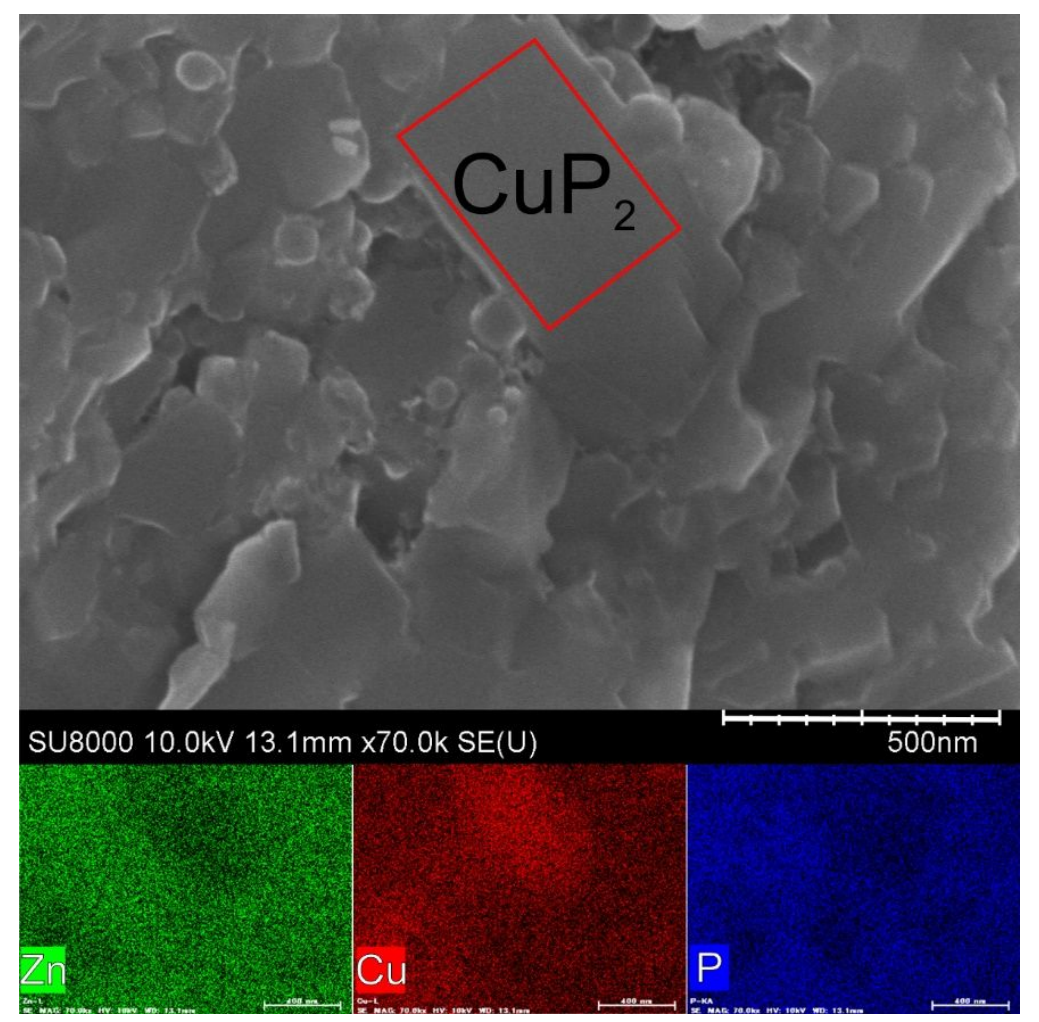

Figure S6. SEM-EDS images highlighting $\mathrm{CuP}_{2}$ impurity particle size. 


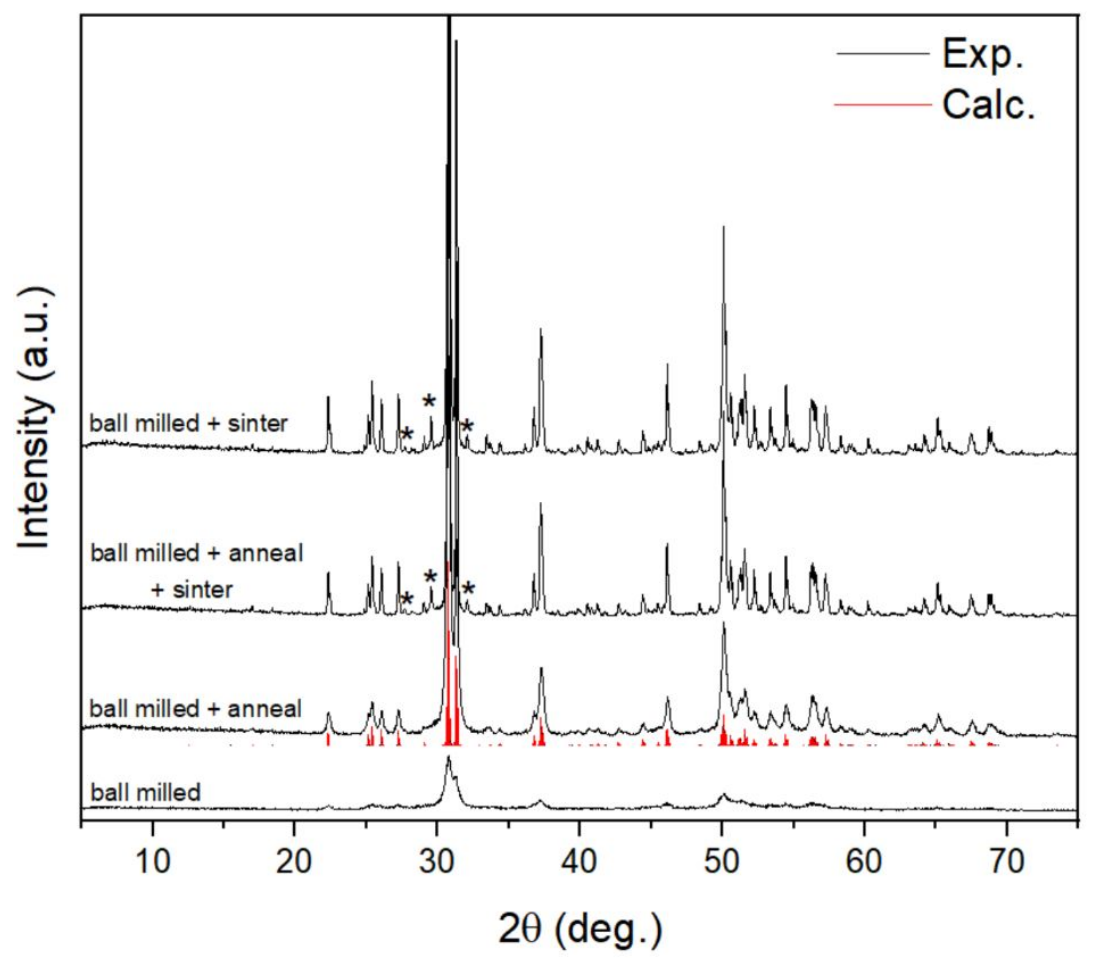

Figure S7. Powder X-ray diffraction patterns of $\mathrm{Zn}_{0.75} \mathrm{Cu}_{2} \mathrm{P}_{8}$ at various stages of synthesis. * denotes unknown impurity peaks. 

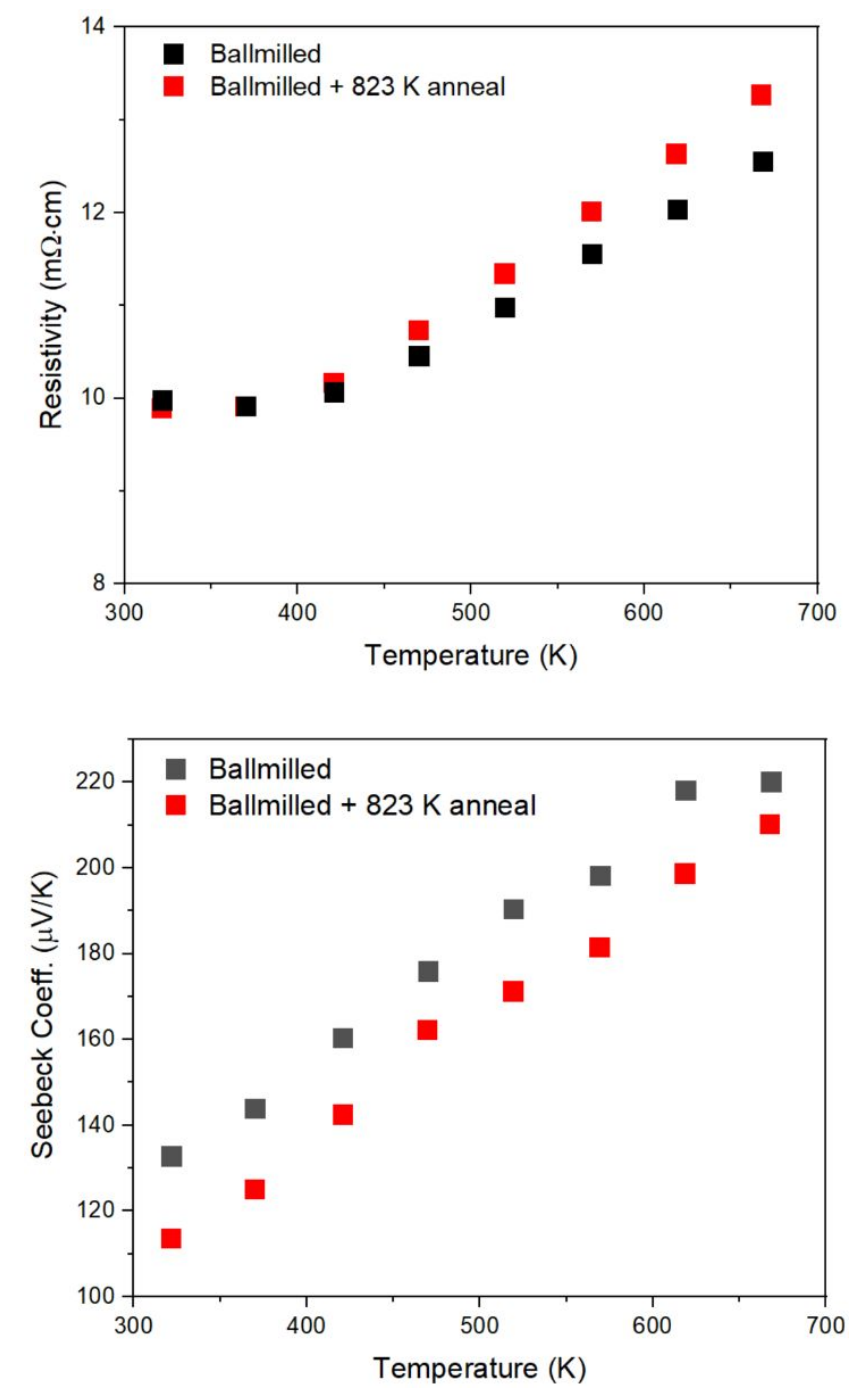

Figure S8. Comparison of electrical resistivity (top) and Seebeck coefficient (bottom) for $\mathrm{Zn}_{0.75} \mathrm{Cu}_{2} \mathrm{P}_{8}$ sintered pellets of an as ball milled sample vs. a ball milled sample annealed at 823 $\mathrm{K}$ for 12 hours prior to sintering.

\section{Synthesis}

The previously reported synthetic method was a high temperature solid state reaction of the elements, however due to the high $\mathrm{P}$ content of $\mathrm{ZnCu}_{2} \mathrm{P}_{8}$ and the volatile/explosive nature of $\mathrm{P}$ in sealed ampoules at elevated temperatures we explored a low temperature synthetic route suitable for larger scale synthesis. ${ }^{1}$ The starting materials, $\mathrm{Zn}$ powder (Wako, 99.9\%), Cu powder (Sigma Aldrich, 99.5\%), and red P powder (Alfa Aesar, 98.9\%) were used as received. In an argon filled glovebox $\left(\mathrm{O}_{2}\right.$ and $\left.\mathrm{H}_{2} \mathrm{O}<3 \mathrm{ppm}\right)$, stoichiometric amounts of the elements were loaded into a stainless-steel ball mill jar with stainless-steel balls (sample-to-ball weight ratio of 1:3) for the target compositions of $\mathrm{Zn}_{1-x} \mathrm{Cu}_{2+x} \mathrm{P}_{8}(x=-0.25,0,0.1,0.25)$ and $\mathrm{Zn}_{0.75} \mathrm{Cu}_{2} \mathrm{P}_{8}$. The jar was sealed and removed from the glovebox. The sample was then ball milled for $12 \mathrm{~h}$ using a SPEX 8000M 
Mixer/Mill. The resulting product is a fine black powder which was determined to match $\mathrm{ZnCu}_{2} \mathrm{P}_{8}$ based on powder X-ray diffraction. The ball milled powders were then sintered into high density pellets for further evaluation (See Spark Plasma Sintering section below). Crystallite sizes achieved for the ball milling procedure are $\sim 10-20 \mathrm{~nm}$, compared to $\sim 45-55 \mathrm{~nm}$ after sintering and mortar and pestle grinding according to the Scherrer equation.

\section{Powder X-ray Diffraction}

Powder X-ray diffraction was performed using a Rigaku SmartLab3 with $\mathrm{Cu}-\mathrm{K}_{\alpha}$ radiation and operating at $40 \mathrm{kV}$ and $40 \mathrm{~mA}$. Sample powder was prepared using a well holder and compressed to a flat surface using a glass slide. Diffraction patterns were collected in the $2 \theta$ range of $5-80^{\circ}$ with a step size of $0.02^{\circ}$ and scan rate of $2^{\circ}$ per minute. Rietveld refinement was performed using the GSAS-II software package. ${ }^{2}$

\section{Spark Plasma Sintering (SPS)}

High density pellets of $10 \mathrm{~mm}$ diameter were sintered using a SPS-1080 (SPS SYNTEX Inc.) Ball milled powder was loaded into a graphite die between graphite foil and graphite plungers. The pellets were sintered under an argon atmosphere by heating to $893 \mathrm{~K}$ over 7 minutes and annealing at this temperature for 10 minutes. The entire sintering procedure was conducted under a uniaxial pressure of $76 \mathrm{MPa}$. After sintering, pellets were removed from the graphite die and polished to remove traces of graphite. The geometrical densities of the resulting pellets were found to be $\geq$ $94 \%$ of their theoretical density.

\section{Energy Dispersive X-ray Spectroscopy (EDS)}

Elemental analysis of samples was performed using a FE-SEM SU8000 (Hitachi) equipped with a Quantax FQ5060 EDS detector (Bruker). Measurements were performed on polished pieces of sintered pellets mounted on carbon tape with the energy of the electron beam held at $10 \mathrm{kV}$. Bulk compositions for each sample were determined by averaging three spots normalized to their respective $\mathrm{Zn}$ content.

\section{UV-Vis Diffuse Reflectance Spectroscopy}

UV-Vis diffuse reflectance spectra were collected in the range of 300-2500 nm using a V-770 UVVis/NIR spectrophotometer equipped with an integrating sphere (JASCO). Pieces of sintered pellets were finely ground into powder with a mortar and pestle and pressed against a glass slide for measurements. Data was then converted using the Kubelka-Munk function to determine the bandgap.

\section{Transport Properties}

Samples were cut into rectangular bars with approximate dimensions of $2 \mathrm{~mm}$ x $2 \mathrm{~mm}$ x $8 \mathrm{~mm}$ for electrical property measurements. Simultaneous electrical resistivity and Seebeck coefficient measurements were performed using a ZEM-2 (ULVAC-Riko) under a helium atmosphere in the temperature range of 323-673 K. Despite the reported high temperature stability of $\mathrm{ZnCu}_{2} \mathrm{P}_{8}$, attempts to measure higher than $673 \mathrm{~K}$ (i.e., $723 \mathrm{~K}$ ) resulted in a reaction between the sample and Ni electrode of the ZEM-2, thus we limit our study to $673 \mathrm{~K} .{ }^{1}$ 
Thermal diffusivity measurements were performed on samples cut into $10 \mathrm{~mm}$ diameter disks of approximately $1.5 \mathrm{~mm}$ thickness. Thermal diffusivity and specific heat were measured under a nitrogen atmosphere using a LFA 467 Hyperflash (NETZSCH) and a Pyroceram standard in the temperature range of 303-673 K. The thermal conductivity $(\kappa)$ was then determined by the equation $\kappa=\rho D C p$ where $\rho, D$, and $C p$ are the sample density, thermal diffusivity, and specific heat, respectively. Three shots were measured at each temperature and the average values are presented in this work.

Transport properties were measured both parallel and perpendicular to the sintering direction of samples, however no significant anisotropic effects were observed.

\section{References}

(1) Nuss, J.; Wedig, U.; Philippi, K.; Takagi, H. Dependence of the Physical Properties on the Cation Ordering in the New Ternary Phosphide $\mathrm{ZnCu}_{2} \mathrm{P}_{8}$. Z. Anorg. Allg. Chem. 2020, 646, 1144-1150.

(2) Toby, B. H.; Von Dreele, R. B., GSAS-II: the genesis of a modern open-source allpurpose crystallography software package. J. Appl. Crystallogr. 2013, 46, 544-549. 